\title{
Usefulness of a Pressure Wire for the Diagnosis of Vasospastic Angina during a Spasm Provocation Test
}

\author{
Teragawa $\mathrm{H}^{*}$, Fujii $\mathrm{Y}^{1}$, Uchimura $\mathrm{Y}^{1}$, Oshita $\mathrm{C}^{1}$, Ueda $\mathrm{T}^{1}$ and Kihara $\mathrm{Y}^{2}$ \\ ${ }^{1}$ Department of Cardiovascular Medicine, JR Hiroshima Hospital, Hiroshima, Japan \\ ${ }^{2}$ Department of Cardiovascular Medicine, Hiroshima University Hospital, Hiroshima, Japan
}

${ }^{*}$ Corresponding author: Teragawa H, Department of Cardiovascular Medicine, JR Hiroshima Hospital, 3-1-36 Futabanosato, Higashi-ku, Hiroshima 732-0057, Japan, Fax: +81-82-262-1499, Tel: +81-82-262-1171, E-mail: hiroteraga71@gmail.com

Citation: Teragawa H, Fujii Y, Uchimura Y, Oshita C, Ueda T, et al. (2017) Usefulness of a Pressure Wire for the Diagnosis of Vasospastic Angina during a Spasm Provocation Test. J Clin Exp Res Cardiol 3(2): 203

Received Date: May 5, 2017 Accepted Date: July 21, 2017 Published Date: July 23, 2017

\begin{abstract}
Objectives: We performed spasm provocation tests (SPTs) using a pressure wire and compared the results with those from patients who underwent the same test without a pressure wire.

Background: The SPT is an important examination used to diagnose vasospastic angina; however, it is sometimes accompanied by severe complications.

Methods: We enrolled 190 patients who underwent an SPT: a pressure wire was used in 103 (Group I) and no wire was used in 87 (Group II). We attempted to place the pressure wire in the distal portions of the right coronary artery and left anterior descending coronary artery. The pressure ratio of the distal lesion to the proximal lesion ( $\mathrm{Pd} / \mathrm{Pa}$ index) was monitored continuously during the test. Vasospastic angina was defined as an angiographic vasoconstriction of the coronary artery accompanied by ischemic chest symptoms and/or electrocardiographic changes. The fluoroscopic time, total dose of contrast medium, and frequency of severe complications were investigated in both groups.

Results: The fluoroscopic time was longer in Group I than in Group II (13.0 $\pm 5.2 \mathrm{~min}$ vs. $11.5 \pm 5.0 \mathrm{~min}, \mathrm{p}=0.0329)$. However, the volume of contrast medium used was lower in Group I (127 \pm 30 and $138 \pm 24 \mathrm{~mL}, \mathrm{p}=0.0058)$, as was the frequency of severe complications ( $2 \%$ vs. $8 \%, \mathrm{p}=0.0484$ ). The minimal $\mathrm{Pd} / \mathrm{Pa}$ index during an SPT was $0.74 \pm 0.15$ in the 119 vessels with spasms and $0.91 \pm 0.07$ in the 76 vessels without spasms. The $\mathrm{Pd} / \mathrm{Pa}$ cut-off value for detecting a coronary spasm was 0.84 , with a sensitivity of $79 \%$ and a specificity of $85 \%$.

Conclusion: The use of a pressure wire during an SPT may help establish a firm diagnosis of vasospastic angina.

Keywords: Vasospastic angina; Coronary spasm; Acetylcholine; Spasm provocation test; Pressure wire; Intracoronary pressure

List of Abbreviations: ACh: Acetylcholine; CAG: Coronary Angiography; ECG: Electrocardiogram; LAD: Left Anterior Descending Coronary Artery; LCA: Left Coronary Artery; LVEF: Left Ventricular Ejection Fraction; NTG: Nitroglycerin; Pd/Pa index, Pressure Ratio of the Distal Lesion to the Proximal Lesion; RCA: Right Coronary Artery; ROC: Receiver Operating Characteristics; SPT: Spasm Provocation Test; VSA: Vasospastic Angina
\end{abstract}

\section{Introduction}

Vasospastic angina (VSA) is characterized by transient narrowing of epicardial coronary arteries and cause myocardial ischemia $[1,2]$. In these cases, a coronary spasm may not only cause angina at rest but also acute myocardial infarction or sudden ischemic cardiac arrest $[1,2]$. Therefore, diagnosing VSA is clinically important.

Although the diagnosis of VSA is usually made by the presence of characteristic chest pains and transient ST-segment changes on electrocardiography (ECG), many patients cannot be diagnosed by ECG changes alone and usually require a spasm provocation test (SPT) [3-5]. Despite being clinically important, this examination can be accompanied by severe complications such as shock, ventricular fibrillation, ventricular tachycardia, and bradyarrhythmia [6-9]. A positive SPT is judged by angiographic confirmation of coronary artery spasm combined with ischemic symptoms and ECG changes [3]. Nevertheless, we sometimes experience cases in which the diagnosis of VSA is difficult because coronary spasms occur without symptoms or ECG changes, because changes are only moderate on angiography, or because symptoms are atypical. Thus, there is a need for an alternative clinical measure of coronary spasm in some patients. 
In cases of moderate organic coronary stenosis, intracoronary pressure assessment with a pressure wire has been widely used in clinical settings, and has not been associated with severe complications [10,11]. Therefore, we assessed the usefulness, safety, and procedure-related parameters associated with using a pressure wire during an SPT and compared the results with those for standard SPT performed without a pressure wire.

\section{Methods}

\section{Study population}

In this observations study, we enrolled 190 patients who underwent coronary angiography (CAG) with an SPT from 2012 to 2015 for the evaluation of chest symptoms that were felt mostly at rest. We excluded patients with moderate chronic kidney disease and worse (estimated glomerular filtration ratio $<45 \mathrm{~mL} / \mathrm{min} / 1.73 \mathrm{~m}^{2}$ ) and significant coronary stenosis $(>75 \%)$ from the study. The protocol was approved by the Ethics Committee of our institution, and written informed consent was obtained from all patients.

From 2012 to mid-2013, we performed SPTs without a pressure wire, and from mid-2013 to 2015 we performed SPTs with a pressure wire. However, we sometimes performed SPT without a pressure wire in the latter period because of wire-related problems or time restrictions. For analysis, the patients were divided into 2 groups according to the use of a pressure wire: Group I included 103 patients who underwent SPTs with a pressure wire and Group II included 87 patients who underwent SPTs without a pressure wire.

\section{Coronary angiography and SPT}

All antianginal agents were discontinued at least $48 \mathrm{~h}$ before catheterization, except for sublingual nitroglycerin (NTG), which was only withheld from $1 \mathrm{~h}$ before catheterization. We performed SPTs after standard diagnostic CAG using the percutaneous brachial approach. A 5-French gage (Fr) transient pacing catheter (Bipolar Balloon Catheter, Bebrawn, Melsungen, Germany) was inserted into the right ventricle via the internal jugular vein or medial cubital vein and set at $50 \mathrm{beats} / \mathrm{min}$. Arterial pressure, heart rate, and electrocardiography (ECG) readings were monitored continuously and recorded using a multichannel recorder (Polygraph 1600; Nihon Electric Corporation, Tokyo, Japan).

After initial CAG, 30 and $50 \mu \mathrm{g}$ doses of acetylcholine (ACh) were infused into the right coronary artery (RCA) for 20 s, with 3 min intervals between consecutive doses. If coronary spasms were expected to occur easily, we used $10 \mu \mathrm{g}$ of ACh for the first dose; this requirement was determined according to the patients' symptoms, including the frequency of chest symptoms and the presence of associated symptoms (e.g., syncope or "cold sweating"). If coronary spasms were not induced by $50 \mu \mathrm{g}$ of ACh, a maximum dose of $80 \mu \mathrm{g}$ was infused into the RCA. CAG was performed immediately after coronary spasms were induced or the maximum ACh infusion finished.

When coronary spasms were provoked but relieved spontaneously, we moved to SPT of the left coronary artery (LCA) without injecting NTG into the RCA. In such cases, after completing SPT of the LCA, we repeated coronary angiograms following an NTG injection into the RCA. When coronary spasms were prolonged or severe enough to cause unstable hemodynamics, $200 \mu \mathrm{g}$ of NTG was administered by intracoronary injection to relieve the coronary spasms. Once the coronary spasms were relieved, final coronary angiograms of the RCA were performed. After intracoronary NTG injection into the RCA, we performed further SPT for the LCA. This was because we have experienced many patients with a positive spasm provocation for the LCA despite receiving intracoronary NTG injections in the RCA. If there was a small RCA on angiograms or if the catheter was not engaged into the ostium of the RCA, we omitted SPT of the RCA.

After the spasm provocation for the RCA, we infused 50 and $100 \mu \mathrm{g}$ doses of ACh into the LCA for $20 \mathrm{~s}$, with 3 min intervals between doses. If coronary spasms were expected to occur easily, we used $20 \mu \mathrm{g}$ of ACh for the first dose in the LCA. If coronary spasms were not induced by $100 \mu \mathrm{g}$ of $\mathrm{ACh}$, a maximum of $200 \mu \mathrm{g}$ was infused into the LCA, with or without 20 , 40 , or $60 \mu \mathrm{g}$ of ergometrine maleate. CAG was performed immediately after coronary spasms were induced or the maximum ACh infusion finished.

After intracoronary injections of $200 \mu \mathrm{g}$ NTG, we performed the final CAG for the LCA. The $80 \mu \mathrm{g}$ dose in the RCA and the 200 $\mu \mathrm{g}$ dose in the LCA were defined as "higher" ACh doses.

Each coronary angiogram was performed using an autoinjector (ZoneMaster, Sheen Man, Osaka) to inject $5 \mathrm{~mL}$ contrast medium at $2.5 \mathrm{~mL} / \mathrm{s}$. If a moderate atherosclerotic lesion was recognized on angiograms, we measured the fractional flow reserve (FFR) by intravenous infusion of adenosine triphosphate to assess the organic stenosis functionality. If unstable hemodynamics occurred (systolic blood pressure $<90 \mathrm{mmHg}$ ) and/or ST-segment elevations continued on ECG during an SPT, we added another intracoronary injection of NTG and maximized the speed of intravenous volume injection. If unstable hemodynamics and STsegment elevations continued despite these countermeasures, we infused small doses $(2-10 \mu \mathrm{g})$ of intracoronary or intravenous adrenaline [9]. 


\section{SPT with a pressure wire}

In Group I, a 0.014-inch pressure wire (PrimeWire Prestige Plus Guide Wire or Verrata Pressure Guide Wire; Volcano Therapeutics Inc., Rancho Cordova, CA, United States) was advanced through a 5-Fr catheter into the distal segments of the RCA and the left anterior descending coronary artery (LAD). Before this, we performed pressure calibration between the tips of the catheters and the pressure wire at the ostium of the coronary arteries (Figure 1). The ratios of distal intracoronary pressure, derived from the pressure wire, to the aortic pressure, derived from the catheter tips ( $\mathrm{Pd} / \mathrm{Pa}$ index), were monitored continuously. The minimal $\mathrm{Pd} /$ $\mathrm{Pa}$ index in response to each $\mathrm{ACh}$ dose was recorded, and when the $\mathrm{Pd} / \mathrm{Pa}$ index reduced during the coronary spasm [12], the values from just before the angiograms were adopted (Figure 2).

A RCA

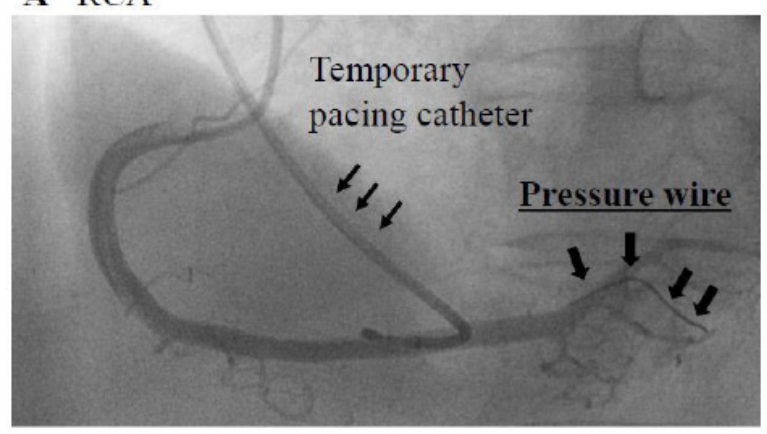

B LAD

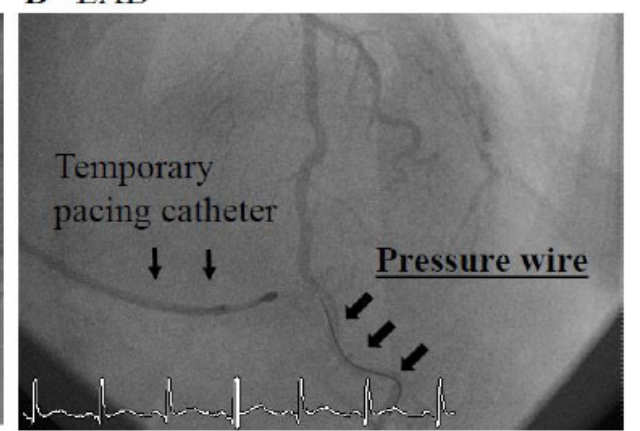

The pressure wire is inserted into the distal portion of the right coronary artery (RCA, A) and distal portion of the left anterior descending coronary artery (LAD, B). The thick arrows indicate the tip of the pressure wire and the thin arrows indicate a temporary pacing catheter. Abbreviations: LAD: left anterior descending coronary artery; RCA: right coronary artery Figure 1: Positions of a pressure wire during spasm provocation testing

A Infusion of $\mathrm{ACh}$

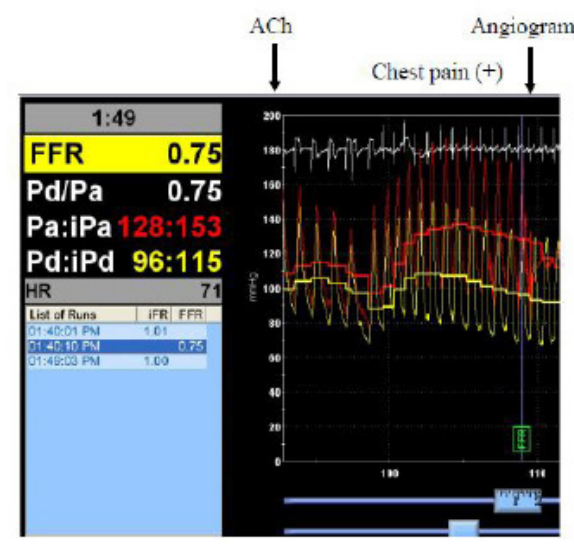

B Infusion of NTG

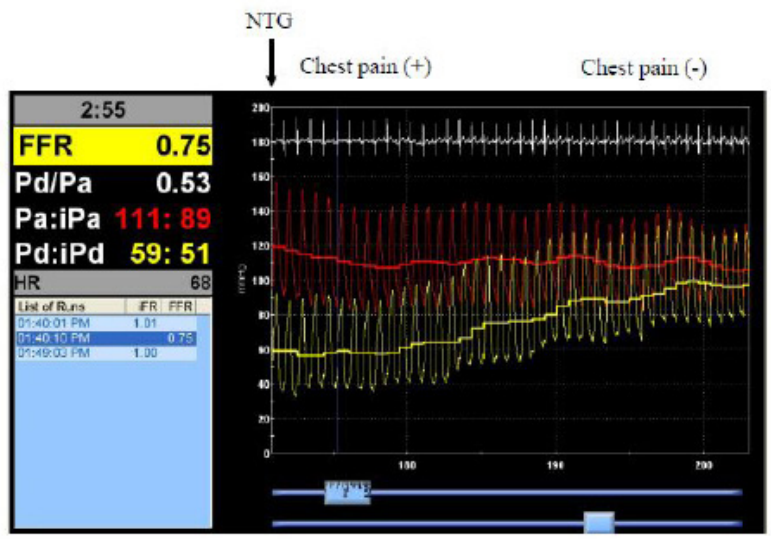

During the SPTs, the Pd/Pa index decreased gradually during a coronary spasm (A). After an intracoronary injection of NTG, the $\mathrm{Pd} / \mathrm{Pa}$ index returned to the baseline value. Abbreviations: ACh: acetylcholine; NTG: nitroglycerin; Pd/Pa index, pressure ratio of the distal lesion to the proximal lesion; SPT: spasm provocation test

Figure 2: A representative case

\section{Quantitative CAG}

The method for measuring coronary artery diameter has been described previously $[13,14]$. We selected spastic and atherosclerotic segments for quantitative analysis. In all cases, the luminal diameters were then measured by a single investigator who was blinded to the clinical data, using an end-diastolic frame in a computer-assisted coronary angiographic analysis system (CAAS II/QUANTCOR; Siemens, Berlin, Germany). Measurements were performed 3 times and the average value was used for analysis. Changes in coronary artery diameter in response to ACh and NTG infusions were expressed as percentage changes from baseline angiographic measurements. The intra-and inter-observer validity of this method are reported to be excellent [15]. Lesions with $>20 \%$ stenosis were defined as atherosclerotic. Angiographic spastic segments were classified by the severity of vasoconstriction as $\geq 90 \%$, subtotal occlusion, and total occlusion.

\section{Definitions of VSA and related parameters}

VSA was defined as a $\geq 90 \%$ narrowing of the epicardial coronary arteries on angiography during SPT, the presence of characteristic chest pain, and/or the presence of ST-segment deviation on ECG [3]. Focal spasm was defined as discrete transient vessel narrowing of $\geq 90 \%$ localized to major or branch coronary arteries. Diffuse spasm was defined as $90 \%$ transient, severe, diffuse vasoconstriction observed in more than 2 adjacent coronary segments of the epicardial coronary arteries and branches [16]. If focal 
spasm occurred in one vessel and a diffuse spasm occurred in another vessel, only focal spasm was assessed for that patient. The presence of multi-vessel spasms was defined as coronary spasms that occurred in more than 2 major coronary branches. We did not assess for the presence of multi-vessel spasms when an SPT was not performed for the RCA because of a small RCA, because we could not engage the catheter into the coronary ostium, or because intracoronary NTG was infused to relieve spasm and the subsequent SPT was negative.

\section{Angiogram-related parameters and SPT-related complications}

The angiogram-related parameters measured in each patient were the total dose of a contrast medium (mL) and the total fluoroscopic time ( $\mathrm{min}$ ). The frequency of FFR measurements was also checked. The frequencies of the following SPT-related complications were measured: paroxysmal atrial fibrillation, prolonged coronary spasm, and prolonged hemodynamic instability, especially requiring the use of adrenaline.

\section{Assessment of biochemical markers and coronary risk factors}

Fasting blood samples were obtained on the day of CAG. Patients were questioned about their smoking status and classified as a current smoker, past smoker (who had stopped smoking for at least 1 month), or nonsmoker. Family history of coronary artery disease was recorded. Blood pressure was measured and hypertension was defined as a systolic blood pressure $\geq 140 \mathrm{mmHg}$, a diastolic blood pressure $\geq 90 \mathrm{mmHg}$, and/or treatment with antihypertensive drugs. Blood chemistry parameters, including total cholesterol, triglycerides, high-density lipoprotein cholesterol, fasting blood sugar, insulin, hemoglobin A1C, and creatinine levels were measured. Low-density lipoprotein cholesterol levels were calculated using the Friedewald equation [17]. Hyperlipidemia was defined as a low-density lipoprotein cholesterol level $\geq 120 \mathrm{mg} / \mathrm{dL}$ and/or treatment with antihyperlipidemic medication. Diabetes mellitus was defined as a fasting blood sugar level $\geq 126 \mathrm{mg} / \mathrm{dL}$, a hemoglobin $\mathrm{A} 1 \mathrm{C} \geq 6.5 \%$, and/or treatment with antidiabetic medication. Finally, the left ventricular ejection fraction (LVEF) was checked.

\section{Statistical analysis}

All data are expressed as mean \pm SD. Baseline characteristics of the 2 groups were compared by Student's unpaired $t$-test or $\chi^{2}$ analysis, as appropriate. The $\mathrm{Pd} / \mathrm{Pa}$ cut-off value for the presence of a coronary spasm was measured by receiver operating characteristics (ROC) analysis. Logistic regression analysis was used to clarify the factors associated with a positive SPT. A $P$-value $<0.05$ was considered statistically significant.

\section{Results}

\section{Patient characteristics, angiogram-related parameters, and complications}

There were 103 patients in Group I and 87 patients in Group II (Table 1), with no difference in characteristics between the 2 groups. However, there were some differences in the angiographic findings and complications (Table 2).

\begin{tabular}{|c|c|c|c|}
\hline & Group I & Group II & p-value \\
\hline Number & 103 & 87 & \\
\hline Age (yrs.) & $67 \pm 11$ & $68 \pm 11$ & 0.4273 \\
\hline Male / Female & $52 / 51$ & $47 / 40$ & 0.6267 \\
\hline Body mass index & $24.2 \pm 4.6$ & $24.7 \pm 4.3$ & 0.5173 \\
\hline Coronary risk factors (\%) & & & \\
\hline Smoking (\%) & $20(19)$ & $17(20)$ & 0.983 \\
\hline Current / Past & $20 / 25$ & $17 / 25$ & 0.6561 \\
\hline Hypertension (\%) & $74(72)$ & $62(72)$ & 0.9296 \\
\hline Lipid disorder (\%) & $65(63)$ & $48(55)$ & 0.2627 \\
\hline Diabetes mellitus (\%) & $22(21)$ & $19(22)$ & 0.903 \\
\hline Family history of CAD (\%) & $25(24)$ & $16(18)$ & 0.3262 \\
\hline Taking statins (\%) & $37(36)$ & $31(36)$ & 0.9668 \\
\hline LVEF on UCG (\%) & $67 \pm 9$ & $66 \pm 10$ & 0.4877 \\
\hline
\end{tabular}

Data were expressed as the mean \pm standard deviation or $\mathrm{n}(\%)$

*Significant at $\mathrm{p}<0.05$

List of abrreviations: CAD, coronary artery disease; LVEF, left ventricular ejection fraction; UCG, echocardiography

Table 1: Patient's characteristics 


\begin{tabular}{|c|c|c|c|}
\hline & Group I & Group II & p-value \\
\hline \multicolumn{4}{|l|}{ Provocations } \\
\hline 1) Use of higher doses of ACh (\%) & $15(15)$ & $3(3)$ & $0.0091^{*}$ \\
\hline 2) Use of addional EM (\%) & $7(7)$ & $5(6)$ & 0.7671 \\
\hline 1) + 2) & $21(20)$ & $8(9)$ & $0.0326^{*}$ \\
\hline Positive SPT (\%) & $78(76)$ & $51(59)$ & $0.0119^{*}$ \\
\hline Diffuse / focal & $57 / 21$ & $41 / 10$ & 0.3417 \\
\hline Multivessel spasm (\%) & $47 / 67(70)$ & $27 / 41(66)$ & 0.6469 \\
\hline Athesclerotic lesions (\%) & $63(61)$ & $52(60)$ & 0.8449 \\
\hline Frequnecy of measuring FFR (\%) & $36(35)$ & $9(10)$ & $<0.0001^{*}$ \\
\hline Total fluoloscopic time (min) & $13.0 \pm 5.2$ & $11.5 \pm 5.0$ & $0.0329^{*}$ \\
\hline $\begin{array}{l}\text { Total volume of contrast medium } \\
\text { ( }(\mathrm{ml})\end{array}$ & $127 \pm 30$ & $138 \pm 24$ & $0.0058^{*}$ \\
\hline Complications (\%) & $14(14)$ & $11(13)$ & 0.8472 \\
\hline 1) Ventricular fibrillation (\%) & $0(0)$ & $1(1)$ & 0.2753 \\
\hline $\begin{array}{l}\text { 2) Hemodynamics instability } \\
\text { requiring adren }\end{array}$ & $2(2)$ & $6(7)$ & 0.0902 \\
\hline 1) +2) & $2(2)$ & $7(8)$ & $0.0484^{*}$ \\
\hline 3) Hemodynamics instablility (\%) & $3(3)$ & $1(1)$ & 0.399 \\
\hline 4) Paroxysmal atrial fibrillation & $10(10)$ & $4(5)$ & 0.1791 \\
\hline
\end{tabular}

Data were expressed as $\mathrm{n}(\%)$ or the mean \pm standard deviation

${ }^{\star}$ Significant at $\mathrm{p}<0.05$

List of abbreviations: ACh, acetylcholine; EM, ergometrine maleate; SPT, spasm provocation test;

FFR, fractional flow reserve

Table 2: Angiographic-related parameters and complications

Regarding provocative drug use, higher ACh doses were used significantly more in Group I ( $\mathrm{p}=0.0091$ ). The frequency of positive SPTs was significantly higher in Group I $(76 \%$ vs. $59 \%, \mathrm{p}=0.0119)$. Although the frequency of atherosclerotic lesions was also similar, the FFR was measured more frequently in Group I ( $p<0.0001)$, which led to a longer total fluoroscopic time in Group I ( $p$ $=0.00329$ ). Among the patients in whom FFR was not measured, the total fluoroscopic time also tended to be longer in Group I ( $\mathrm{n}$ $=67,12.7 \pm 4.4 \mathrm{~min})$ compared with Group II $(\mathrm{n}=78,11.3 \pm 5.1 \mathrm{~min})(\mathrm{p}=0.0935)$. By contrast, the volume of contrast medium was smaller in Group I ( $\mathrm{p}=0.0058)$.

Regarding SPT-related complications, the frequency of hemodynamic instability requiring adrenaline tended to be lower in Group I $(\mathrm{p}=0.0902)$. In addition, the frequency of severe complications, including ventricular fibrillation and hemodynamic instability requiring adrenaline, were lower in Group I $(\mathrm{p}=0.0484)$.

\section{SPT using a pressure wire}

Insertion of the pressure wire itself did not cause vascular trauma or wire-induced coronary spasm. SPT was only attempted in 96 RCA vessels because RCAs were small in 5 patients and we could not engage the catheter in the RCA ostium in 2 patients. The pressure wires were successfully inserted into the distal RCAs in 94 of these 96 vessels (98\%). SPT was attempted in 103 LCA vessels and was successfully inserted into the distal LAD in 101 (98\%). Pd/Pa index monitoring was done for $101 \mathrm{LAD}$ vessels because the pressure wire could not be placed within the LAD in 2 patients.

Of the 195 vessels in which a pressure wire could be inserted distally, the SPT was positive in 119 (61\%) and negative in 76 (39\%). Of the positive cases, 54 were in RCA vessels (57\%) and 65 were in LAD vessels (64\%). Coronary spasm was induced at low ACh doses in 43 vessels (36\%), at high ACh doses in 72 vessels (61\%), and at higher ACh doses (or with additional ergometrine maleate) in 4 vessels (3\%). Angiographic narrowing of spastic segments was $\geq 90 \%$ in 88 lesions (74\%), subtotal occlusion in 23 lesions (19\%), and total occlusion in 8 segments (7\%).

\section{Intracoronary pressure cut-off value for the detection of a coronary spasm}

The minimum $\mathrm{Pd} / \mathrm{Pa}$ indexes during SPT were $0.74 \pm 0.15$ in the 119 spastic vessels and $0.91 \pm 0.07$ in the 76 non-spastic vessels (Figure 3, p < 0.0001). The ROC analysis showed that the optimal cut-off value for the presence of a coronary spasm was 0.84 , and that this had a sensitivity and specificity of $78 \%$ and $84 \%$, respectively (area under the ROC curve, 0.878 ). The $\mathrm{Pd} / \mathrm{Pa}$ index was $0.77 \pm 0.12$ in spastic vessels with $\geq 90 \%$ narrowing, $0.70 \pm 0.15$ in the spastic vessels with subtotal occlusion, and $0.54 \pm 0.25$ in the spastic vessels with total occlusion $(\mathrm{p}<0.0001)$. More RCA vessels $(n=18)$ than LAD vessels $(n=8)$ had a Pd/Pa index $>0.84$, whereas all non-spastic vessels with a $\mathrm{Pd} / \mathrm{Pa}$ index $<0.84$ were recognized in $\mathrm{LAD}$ vessels $(\mathrm{n}=11)$. 


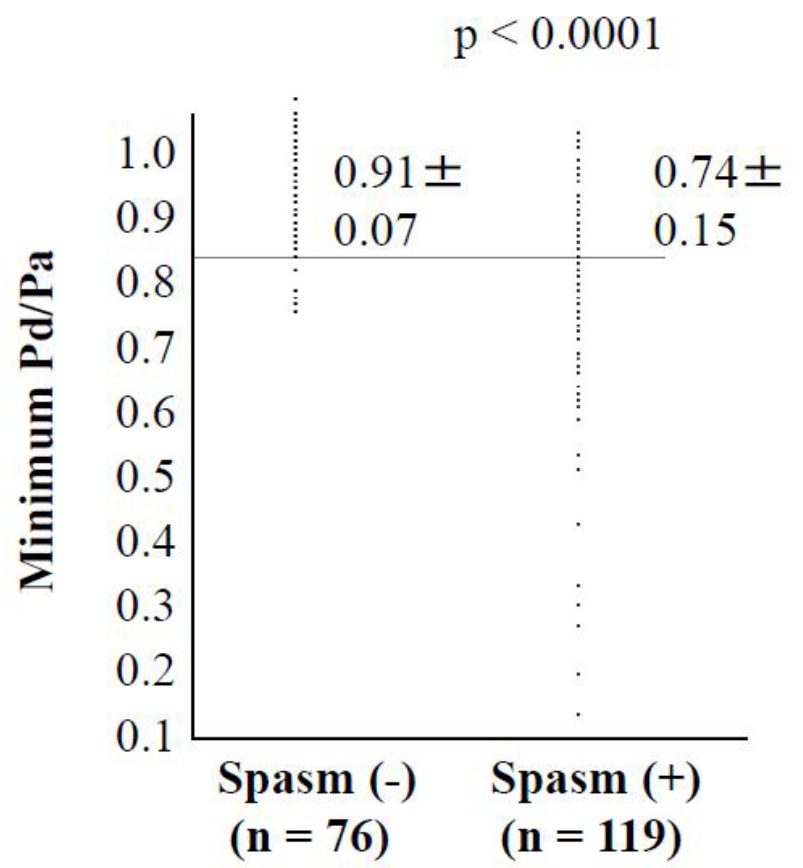

The minimum $\mathrm{Pd} / \mathrm{Pa}$ during an SPT was $0.74 \pm 0.15$ in the spastic vessels $(\mathrm{n}=119)$ and $0.91 \pm$ 0.07 in the non-spastic vessels $(n=76)(p<0.0001)$. Based on the ROC analysis, the cut-off value for the presence of a coronary spasm was 0.84 , with a sensitivity of 0.78 and a specificity of 0.84 . Abbreviations: $\mathrm{Pd} / \mathrm{Pa}$ index, pressure ratio of the distal lesion to the proximal lesion; ROC: receiver operating characteristics; SPT: spasm provocation test

Figure 3: Minimal $\mathrm{Pd} / \mathrm{Pa}$ index during the SPT in spastic and non-spastic vessels

\section{Discussion}

We showed that using a pressure wire during SPT was associated with reductions in the total dose of contrast medium and the frequency of severe complications. Furthermore, we showed that the pressure wire during SPT provided additional information that could be useful when diagnosing VSA.

An SPT is an important clinical diagnostic tool for VSA, and remains so despite reports of severe complications [3,6-9]. These complications may be caused by prolonged hemodynamic instability, severe and prolonged myocardial ischemia, or NTG overdose. Accompanied chest symptoms and ST-segment changes during an SPT are generally reliable indices of myocardial ischemia, but are not always accompanied by a positive SPT. Therefore, to improve the safety and convenience of SPT, indices other than chest symptoms and ECG changes need to be developed to facilitate the prompt detection of myocardial ischemia and recovery.

The usefulness of pressure wires has been established for the functional assessment of organic coronary stenosis, and they are used without severe complications in many clinical settings [10,11]. Consistent with this, pressure wire use did not cause any complications, including coronary spasm, in this study. Naturally, a pressure wire should be inserted into the coronary artery more carefully and slowly during an SPT to avoid guidewire-induced coronary spasm. In this study, using a pressure wire was associated with reductions in both the total contrast medium dose and the frequency of severe complications.

When using a pressure wire during an SPT, it is important to monitor the $\mathrm{Pd} / \mathrm{Pa}$ index continuously. If chest symptoms, ECG changes, and a reduced $\mathrm{Pd} / \mathrm{Pa}$ index were absent, we moved to the next provocation with an increased ACh dose, without performing further angiograms. If the $\mathrm{Pd} / \mathrm{Pa}$ index decreased gradually during the $\mathrm{SPT}$, the occurrence of coronary spasm was anticipated. Furthermore, if a coronary spasm occurred with the reduction in the $\mathrm{Pd} / \mathrm{Pa}$ index, an intracoronary infusion of NTG was given to relieve the spasm and promptly elevate the $\mathrm{Pd} / \mathrm{Pa}$ index to the baseline level. If the $\mathrm{Pd} / \mathrm{Pa}$ index increased after an NTG injection, we waited without an additional angiogram or NTG injection. An SPT using a pressure wire can, therefore, promptly detect myocardial ischemia and its recovery. Indeed, given the process of myocardial ischemia, a change in the $\mathrm{Pd} / \mathrm{Pa}$ index may be the earliest marker of myocardial ischemia, preceding even ECG changes. This, in turn, may have contributed to the reductions seen in the total contrast medium dose and the frequency of severe complications.

In the present study, the frequency of SPT positivity was higher in Group I. A positive SPT was defined as the presence of angiographic vasospasm accompanied by ischemic findings [3], and was not judged based on the $\mathrm{Pd} / \mathrm{Pa}$ index. The insertion of a pressure wire may allow the catheter to engage into the ostium of the coronary artery, especially in the RCA, thereby increasing the ACh dose delivered into that artery. But, this may not be the main cause for the higher frequency of positive SPTs. In our study, the period of pressure wire use (Group I) followed the period without pressure wire use (Group II). Although the patient characteristics were not different between the groups, in the latter period we may have judged patients with a lower likelihood of VSA as inappropriate for SPT and patients with a higher likelihood of VSA as suitable for SPT. 
Recently, the usefulness of higher ACh doses in SPTs has been reported $[5,18,19]$. Since these reports, SPTs using higher ACh doses have been done more frequently, even at our institution. It may be that the higher ACh doses contributed to the differences in the frequency of positive SPT results. Despite adopting higher ACh doses in Group I, reductions in the total contrast medium dose and the frequency of severe complications may be important advantages of SPT with a pressure wire. In addition, the total fluoroscopic time was longer in Group I because of both the time needed to insert the pressure wire and the higher frequency of FFR measurement.

The ROC analysis showed that the Pd/Pa cut-off value for detecting the occurrence of a coronary spasm during an SPT was 0.84, which is similar to the FFR of 0.80 reported for detecting myocardial ischemia [11]. This may therefore be an acceptable value. However, the $\mathrm{Pd} / \mathrm{Pa}$ index should be interpreted carefully because the RCA vessel had a higher index $(>0.84)$ more frequently than the LAD (RCA: 18, LAD: 8), and because coronary spasm occurred at the distal segments of all RCA vessels. Thus, SPT using a pressure wire may be weak in the spasm, which occured at the distal RCA. When the pressure wire was inserted into one branch of the RCA, but coronary spasm occurred at another branch, the Pd/Pd index was not reduced. In addition, 11 non-spastic vessels with a lower $\mathrm{Pd} / \mathrm{Pa}$ index were all in the $\mathrm{LAD}$. In general, the baseline $\mathrm{Pd} / \mathrm{Pa}$ index in the $\mathrm{LAD}$ vessel was lower than that in the RCA because of its anatomical position. Thus, the $\mathrm{Pd} / \mathrm{Pa}$ index in the LAD may be reduced by ACh infusions in the absence of significant coronary spasms.

Although the presence of silent VSA is not described in relevant guidance, we found that some vessels judged as non-spastic based on the absence of ischemic symptoms and ECG changes actually showed angiographic evidence of vasoconstriction. These findings suggest the possibility of silent VSA. However, we did not check the lactate concentration in the coronary sinus during SPT $[3,20]$. If a relationship between the $\mathrm{Pd} / \mathrm{Pa}$ index and myocardial lactate concentration can be clarified, it may be possible to confirm the presence of silent VSA in the future.

An SPT using a pressure wire undoubtedly has higher costs and requires a longer fluoroscopic time than the usual procedure. In addition, the pressure wire cannot detect coronary spasm in coronary branches in which the wire is not inserted, such as the distal branches of the RCA or the left circumflex coronary artery. However, the procedure can promptly detect coronary spasm and its recovery, and may be associated with fewer severe complications. Given the costs, though, it may be best to reserve the procedure for use in the following situations: 1) when hemodynamic instability may be precipitated by coronary spasm, such as when patients have hypertrophic cardiomyopathy or left ventricular dysfunction; 2) when patients have chronic kidney disease; and 3) when cardiologists seek to clarify the disease status through a second SPT [21-24]. In addition to showing that the Pd/Pa index can be measured using $\mathrm{ACh}$ as a provocation drug, we have data showing a comparable response to ergometrine maleate (not shown). The $\mathrm{Pd} / \mathrm{Pa}$ index could, therefore, be relevant when used with different provocation drugs.

\section{Limitations}

There were several limitations to the present study. First, this study was observational and not randomized, and the SPT was not performed at the same time in the 2 groups. SPT using a pressure wire was associated with higher ACh doses at that time, and it is possible that this combination caused the better SPT results in the second period. However, other clinical factors, such as patient characteristics and the SPT protocol (excluding the differences in pressure wire use and the higher ACh doses), were similar between the periods, increasing the likelihood our comparison is meaningful.

Second, our SPT deviated in terms of the testing order (i.e., RCA first, LCA second) and the ACh doses ( 30 and $50 \mu \mathrm{g}$ for the RCA and 50 and $100 \mu \mathrm{g}$ for the LCA). At the $30 \mu \mathrm{g}$ dose in the RCA and the $50 \mu \mathrm{g}$ in the LCA, we skipped the angiograms if patients had no chest symptoms or ST-segments changes on ECG. This method has been used at our institution for some time, and was familiar to the cardiac catheterization staff. The maximum ACh doses in our method were no different to those used in standard methods, so the $\mathrm{Pd} / \mathrm{Pa}$ index may not be different during coronary spasm when comparing the methods. However, the different method of SPT may cause differences in the frequency of multi-vessel spasm, total fluoroscopic time, and total contrast medium dose.

There are other important limitations. For example, FFR was measured more often in patients who underwent SPT with a pressure wire. However, we conclude that this resulted from using a pressure wire, with the frequency of atherosclerotic lesions being comparable between the groups. Another limitation is that we did not investigate the association between values obtained from pressure wires and the lactate concentration in the coronary sinus, which is a reliable marker of myocardial ischemia. We also showed that the $\mathrm{Pd} / \mathrm{Pa}$ index decreased promptly during a coronary spasm and that the minimal $\mathrm{Pd} / \mathrm{Pa}$ index could not be easily determined in some patients. Indeed, the $\mathrm{Pd} / \mathrm{Pa}$ index instantly decreased to $0.2-0.3$ in some patients, and had we waited longer, the minimal $\mathrm{Pd} / \mathrm{Pa}$ index could have been even lowed. The $\mathrm{Pd} / \mathrm{Pa}$ index was measured just before the angiograms to obtain the minimal value. Finally, we adopted the 5-Fr coronary catheters in this study, and it is possible that using the recommended 6-Fr catheters may produce slightly different $\mathrm{Pd} / \mathrm{Pa}$ results.

\section{Conclusion}

We conclude that using a pressure wire during an SPT may reduce the total dose of contrast medium and the frequency of severe complications by improving the detection of myocardial ischemia and its recovery. This procedure can also provide valuable data for use when diagnosing VSA, making it a useful adjunct to SPT in specific cases. 


\section{Acknowledgment}

The authors acknowledge all staff at the catheterization room.

\section{References}

1. Yasue H, Kugiyama K (1997) Coronary spasm: clinical features and pathogenesis. Intern Med 36: 760-5.

2. Yasue H, Nakagawa H, Itoh T, Harada E, Mizuno Y (2008) Coronary artery spasm--clinical features, diagnosis, pathogenesis, and treatment. J Cardiol 51: 2-17.

3. JCS Joint Working Group (2014) Guidelines for diagnosis and treatment of patients with vasospastic angina (Coronary Spastic Angina) (JCS 2013). Circ J 78: 2779-801.

4. Sueda S, Kohno H, Fukuda H, Ochi N, Kawada H, et al. (2003) Induction of coronary artery spasm by two pharmacological agents: comparison between intracoronary injection of acetylcholine and ergonovine. Coron Artery Dis 14: 451-7.

5. Sueda S, Kohno H, Ochi T, Uraoka T (2015) Overview of the Acetylcholine Spasm Provocation Test. Clin Cardiol 38: 430-8.

6. Sueda S, Kohno H, Fukuda H, Ochi N, Kawada H, Hayashi Y, et al. (2004) Frequency of provoked coronary spasms in patients undergoing coronary arteriography using a spasm provocation test via intracoronary administration of ergonovine. Angiology 55: 403-11.

7. Takagi Y, Yasuda S, Takahashi J, Tsunoda R, Ogata Y, et al. (2013) Clinical implications of provocation tests for coronary artery spasm: safety, arrhythmic complications, and prognostic impact: multicentre registry study of the Japanese Coronary Spasm Association. Eur Heart J 34: 258-67.

8. Sueda S, Kohno H (2016) Overview of complications during pharmacological spasm provocation tests. J Cardiol 68: 1-6.

9. Teragawa H, Nishioka K, Higashi Y, Chayama K, Kihara Y (2008) Treatment of Coronary Spastic Angina, Particularly Medically Refractory Coronary Spasm. Clin Med (Cardiology) 2: 181-89.

10. De Bruyne B1, Fearon WF, Pijls NH, Barbato E, Tonino P, et al. (2014) Fractional flow reserve-guided PCI for stable coronary artery disease. N Engl J Med 371: 1208-17.

11. Windecker S, Kolh P, Alfonso F, Collet JP, Cremer J, et al. (2014) 2014 ESC/EACTS Guidelines on myocardial revascularization: The Task Force on Myocardial Revascularization of the European Society of Cardiology (ESC) and the European Association for Cardio-Thoracic Surgery (EACTS)Developed with the special contribution of the European Association of Percutaneous Cardiovascular Interventions (EAPCI). Eur Heart J 35: 2541-619.

12. Teragawa H, Fujii Y, Ueda T, Murata D, Nomura S (2015) Case of angina pectoris at rest and during effort due to coronary spasm and myocardial bridging. World J Cardiol 7: 367-72.

13. Teragawa H, Mitsuba N, Ishibashi K, Kurisu S, Kihara Y (2013) Positive influence of aspirin on coronary endothelial function: Importance of the dose. World J Cardiol 5: 426-33.

14. Teragawa H, Mitsuba N, Ishibashi K, Nishioka K, Kurisu S, et al. (2013) Evaluation of coronary microvascular function in patients with vasospastic angina. World J Cardiol 5: 1-7.

15. Teragawa H, Kato M, Yamagata T, Matsuura H, Kajiyama G (2001) Magnesium causes nitric oxide independent coronary artery vasodilation in humans. Heart 86: $212-6$.

16. Sato K, Kaikita K, Nakayama N, Horio E, Yoshimura H, et al. (2013) Coronary vasomotor response to intracoronary acetylcholine injection, clinical features, and long-term prognosis in 873 consecutive patients with coronary spasm: analysis of a single-center study over 20 years. J Am Heart Assoc 2: e000227.

17. Friedewald WT, Levy RI, Fredrickson DS (1972) Estimation of the concentration of low-density lipoprotein cholesterol in plasma, without use of the preparative ultracentrifuge. Clin Chem 18: 499-502.

18. Sueda S, Kohno H, Miyoshi T, Sakaue T, Sasaki Y, et al. (2015) Maximal acetylcholine dose of $200 \mu \mathrm{g}$ into the left coronary artery as a spasm provocation test: comparison with $100 \mu \mathrm{g}$ of acetylcholine. Heart Vessels 30: 771-8.

19. Ong P, Athanasiadis A, Borgulya G, Vokshi I, Bastiaenen R, et al. (2014) Clinical usefulness, angiographic characteristics, and safety evaluation of intracoronary acetylcholine provocation testing among 921 consecutive white patients with unobstructed coronary arteries. Circulation 129: 1723-30.

20. Kaikita K, Ishii M, Sato K, Nakayama M, Arima Y, et al. (2015) Determinants of Myocardial Lactate Production During Acetylcholine Provocation Test in Patients With Coronary Spasm. J Am Heart Assoc 4: e002387.

21. Sueda S, Ochi N, Kawada H, Matsuda S, Hayashi Y, et al. (1999) Frequency of provoked coronary vasospasm in patients undergoing coronary arteriography with spasm provocation test of acetylcholine. Am J Cardiol 83: 1186-90.

22. Sueda S, Kohno H, Oshita A, Izoe Y, Nomoto T, et al. (2009) Vasospastic heart failure: multiple spasm may cause transient heart failure? J Cardiol 54: 452-9.

23. Inami T, Kataoka M, Shimura N, Ishiguro H, Kohshoh H, et al. (2012) Left ventricular dysfunction due to diffuse multiple vessel coronary artery spasm can be concealed in dilated cardiomyopathy. Eur J Heart Fail 14: 1130-8.

24. Teragawa H, Fujii Y, Uchimura Y, Ueda T (2017) Importance of a second spasm provocation test: Four cases with an initial negative spasm provocation test. World J Cardiol 9: 289-95. 


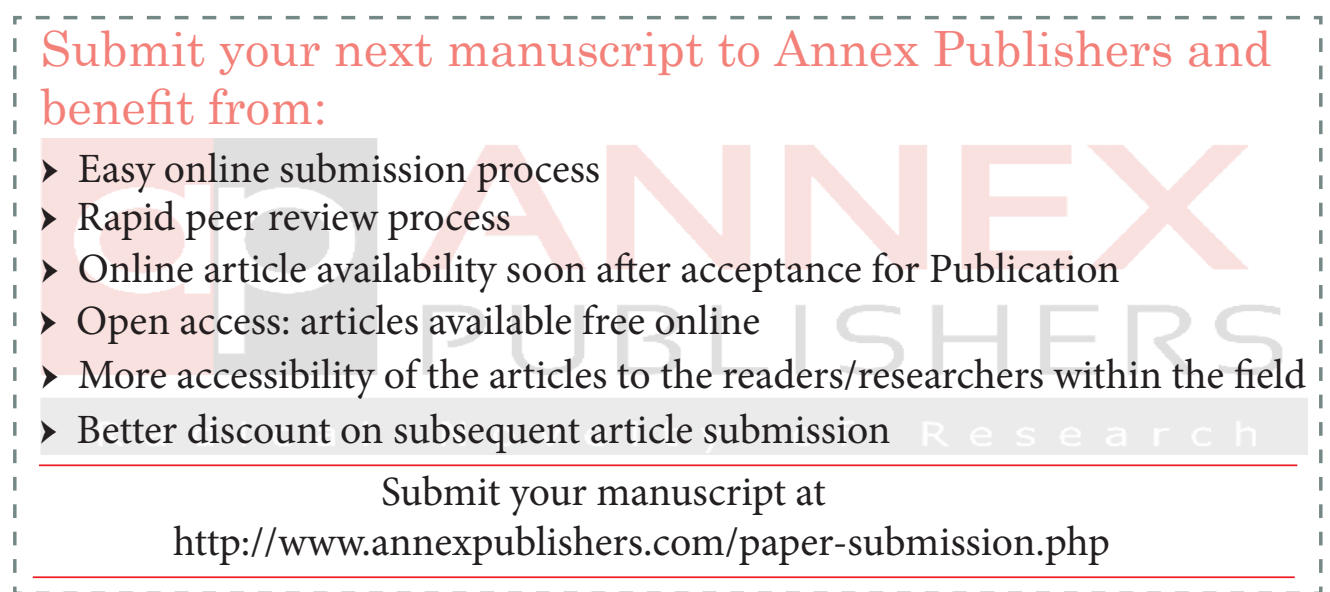

